\title{
On Non-Complete Sets and Restivo's Conjecture
}

\author{
Vladimir V. Gusev, Elena V. Pribavkina \\ Ural State University, Lenina st. 51, 620083, Ekaterinburg, Russia \\ vl.gusev@gmail.com, elena.pribavkina@usu.ru
}

\begin{abstract}
A finite set $S$ of words over the alphabet $\Sigma$ is called noncomplete if $\operatorname{Fact}\left(S^{*}\right) \neq \Sigma^{*}$. A word $w \in \Sigma^{*} \backslash \operatorname{Fact}\left(S^{*}\right)$ is said to be uncompletable. We present a series of non-complete sets $S_{k}$ whose minimal uncompletable words have length $5 k^{2}-17 k+13$, where $k \geq 4$ is the maximal length of words in $S_{k}$. This is an infinite series of counterexamples to Restivo's conjecture, which states that any non-complete set possesses an uncompletable word of length at most $2 k^{2}$.
\end{abstract}

\section{Introduction}

Let $\Sigma$ be a finite alphabet. A finite set $S$ of words over the alphabet $\Sigma$ is called complete if $\operatorname{Fact}\left(S^{*}\right)=\Sigma^{*}$, i.e. every word over the alphabet $\Sigma$ is a factor of a word of $S^{*}$. If $S$ is not complete, $\Sigma^{*} \backslash \operatorname{Fact}\left(S^{*}\right)$ is not empty and a word in this set of minimal length is called a minimal uncompletable word (with respect to the non-complete set $S$ ). Its length will be denoted by uwl(S).

The problem of finding minimal uncompletable words and their length was introduced in 1981 by Restivo. In his paper [5] he conjectured that a noncomplete set $S$ always possesses an uncompletable word $w$ of length at most $2 k^{2}$, where $k$ is the maximal length of words in $S$, and $w$ is of the form $w=$ $u v_{1} u v_{2} \cdots u v_{k-1} u$, where $u \notin S,|u|=k$ and $\left|v_{i}\right| \leq k$ for all $i=1,2, \ldots, k-1$. An example giving a lower bound $k^{2}+k-1$ for the length of minimal uncompletable words was presented in [3. However Restivo's conjecture appeared to be false by means of a counterexample found in [2]. Namely, let $k>6$ and let $R_{k}=\Sigma^{k} \backslash\left\{a^{k-2} b b\right\} \cup \Sigma b a^{k-4} \Sigma \cup \Sigma b a \cup b^{4} \cup J_{k}$, where $J_{k}=\bigcup_{i=1}^{k-3}\left(b a^{i} \Sigma \cup a^{i} b\right)$. In 2] the authors computed for $7 \leq k \leq 12$ that the length of a minimal uncompletable word for $R_{k}$ is equal to $3 k^{2}-9 k+1$ but were unable to prove it in general.

In this paper we present a new series of non-complete sets $S_{k}$ whose minimal uncompletable words have length $5 k^{2}-17 k+13$ for $k \geq 4$.

As far as the upper bound is concerned, only trivial exponential one is known. More precisely, the length of a minimal uncompletable word is at most $2^{\|S\|-m+1}$, where $m$ is the number of elements in $S$ and $\|S\|$ is the sum of lengths of all elements in $S$. It comes from the connection between non-complete sets and synchronizing automata studied in [3]. However this bound is not likely to be precise.

An interesting related question of deciding whether a given regular language $L$ satisfies one of the properties $\Sigma^{*}=\operatorname{Fact}(L), \Sigma^{*}=\operatorname{Pref}(L), \Sigma^{*}=\operatorname{Suff}(L)$ has 
been recently considered by Rampersad et al. in [4, where the computational complexity of the aforementioned problems in case $L$ is represented by a deterministic or non-deterministic finite automaton is studied. In particular case $L=S^{*}$ for $S$ being a finite set of words the authors mention that the complexity of deciding whether or not $\Sigma^{*}=\operatorname{Fact}\left(S^{*}\right)$ is still an open problem.

\section{The set $S_{k}$}

To fix the notation, let us recall some basic definitions from combinatorics on words. By $|w|$ we denote the length of a word $w$. The length of the empty word $\varepsilon$ is equal to zero. By $\Sigma^{+}$we denote the set of all non-empty words over the alphabet $\Sigma$; by $\Sigma^{k}$ - the set of all words of length $k$ over $\Sigma$ and by $\Sigma \leq k-$ the set of all words of length at most $k$ over $\Sigma$. A word $u \in \Sigma^{+}$is a factor of $w$ (prefix or suffix respectively) if $w$ can be decomposed as $w=x u y(w=u y$ or $w=x u$ respectively) for some $x, y \in \Sigma^{*}$. A factor (prefix, suffix) $u$ of $w$ is called proper if $u \neq w$. Given a word $u=a_{1} a_{2} \cdots a_{n} \in \Sigma^{+}$by $u[i \ldots j]$ with $1 \leq i, j \leq n$ we denote the factor $a_{i} a_{i+1} \cdots a_{j}$ if $i \leq j$, and the empty word if $i>j$. Moreover, we put $u[0]=\varepsilon$.

Let $\Sigma=\{a, b\}$. Consider the set

$$
S_{k}=\left(\Sigma^{k} \backslash\left\{b a^{k-1}, b^{k-1} a\right\}\right) \cup\left(\Sigma^{k-1} \backslash\left\{a^{k-1}, b^{k-1}\right\}\right) .
$$

In section 3 we show that this set is not complete for $k \geq 4$ and possesses an uncompletable word of length $5 k^{2}-17 k+13$. In section 4 we show that this upper bound is precise. Our results considerably rely upon the notion of a forbidden position in a word. This notion was introduced in [3. Let $S$ be any non-complete set and let $w$ be an uncompletable word for the set $S$. We say that $0 \leq j \leq|w|-1$ is a forbidden position in $w$ with respect to $S$, if $w[j+1, \ldots,|w|] \notin \operatorname{Pref}\left(S^{*}\right)$, i.e. the suffix of the word $w$ starting from position $j$ is not a prefix of any word in $S^{*}$. If the set $S$ is clear from context we will omit reference to $S$. Note that, if $S \subseteq \Sigma^{\leq k}$ and positions $0,1, \ldots, k-1$ are forbidden in some word $w$, then $w$ is uncompletable for $S$. So to prove that a set $S \subseteq \Sigma^{\leq k}$ is not complete, it is enough to find a word with first $k$ forbidden positions.

Lemma 1. Let $j \geq k$ be a forbidden position in a word $w$ with respect to $S_{k}$. Then the position $j-k$ is forbidden in $w$ with respect to $S_{k}$ iff either $j-1$ is forbidden or $w[j-k+1, \ldots, j-1] \in\left\{a^{k-1}, b^{k-1}\right\}$.

Proof. Let $j-k$ be forbidden in $w$. Then by definition $w[j-k+1, \ldots,|w|] \notin$ $\operatorname{Pref}\left(S_{k}^{*}\right)$. Suppose $j-1$ is not forbidden in $w$, i.e. $w[j, \ldots,|w|]=x \in \operatorname{Pref}\left(S_{k}^{*}\right)$. If the factor $y=w[j-k+1, \ldots, j-1]$ of length $k-1$ is in $S_{k}$, then $w[j-k+$ $1, \ldots,|w|]=y x \in \operatorname{Pref}\left(S_{k}^{*}\right)$, which is a contradiction. Hence $y \in \Sigma^{k-1} \backslash S_{k}=$ $\left\{a^{k-1}, b^{k-1}\right\}$.

Conversely, arguing by contradiction suppose $j-k$ is not forbidden. Then since the length of the suffix $w[j-k+1, \ldots,|w|]$ is at least $k$, it can be factorized as $x y$ where $x \in S_{k}$ and $y \in \operatorname{Pref}\left(S_{k}^{*}\right)$. The case $|x|=k$ contradicts the condition 
that $j$ is forbidden, since we get $w[j+1, \ldots,|w|]=y \in \operatorname{Pref}\left(S_{k}^{*}\right)$. Hence $|x|=k-1$ and since $x \in S_{k}$ we have that $x$ is different both from $a^{k-1}$ and $b^{k-1}$. But then position $j-1$ is not forbidden, which is a contradiction.

In the rest of the paper we strictly fix the following notation: $u=b a^{k-1}$ and $v=b^{k-1} a$. We will consider forbidden positions only in occurrences of $u$ and $v$ in $w$. In each such occurrence for convenience we will enumerate forbidden positions locally from 0 to $k-1$.

Example 1. Consider the following word:

$$
w=\underbrace{\prime}_{\{0,1,2\}} \underbrace{b^{\prime} a^{\prime} a}_{\{0,2\}} a^{\prime} b^{\prime} \underbrace{\{1\}}_{\{0\}} \overbrace{b^{\prime} a}^{\{1} a \underbrace{\prime b b a} .
$$

Using definition and lemma 1 it is easy to calculate the set of its forbidden positions with respect to $S_{3}:\{0,1,2,4,5,7,10\}$. There are two occurrences of $u$ and two occurrences of $v$ in $w$ (the first occurrence of $v$ overlaps with the second occurrence of $u$ ). Locally enumerated sets of forbidden positions are: $\{0,1,2\}$ in the first occurrence of $u,\{1\}$ in the second occurrence of $u,\{0,2\}$ in the first occurrence of $v$, and $\{0\}$ in the second occurrence of $v$. Note that, since first three positions are forbidden in $w$, this word is uncompletable for $S_{3}$.

Position 0 may be forbidden in an occurrence of $u$ or $v$. The following statement gives necessary and sufficient conditions for this to happen. It is an easy consequence of lemma 1 .

Lemma 2. Position 0 is forbidden in an occurrence of $u$ in a word $w$ iff position $k-1$ is forbidden in the same occurrence of $u$. Position 0 is forbidden in any occurrence of $v$ in $w$.

Two occurrences $p, q \in\{u, v\}$ in a word $w$ are said to be consecutive if they either overlap or are the only occurrences from $\{u, v\}$ in the factor $p x q$ of $w$.

Lemma 3. Let $p, q \in\{u, v\}$ be two consecutive occurrences without overlap in a word $w$, and let $p x q$ be a factor of $w$ with $|x| \geq 0$. Let $F_{p}$ and $F_{q}$ be the sets of forbidden positions in $p$ and $q$ respectively. Then

$$
F_{p} \subseteq\left\{j+|x| \bmod k \mid j \in F_{q}\right\} \cup\{0\}
$$

Proof. Consider a forbidden position $i$ in $p$ such that $i \neq 0$ and consider the factor $y$ of length multiple of $k$ in $w$ from position $i$ in $p$ to some position $j$ in $q$ $(0 \leq j<k)$. This factor is in $S_{k}^{+}$, since $p$ and $q$ are consecutive occurrences of words from $\Sigma^{k} \backslash S_{k}$. Thus, if position $j \notin F_{q}$, then neither position $i$ is forbidden. On the one hand, we have $|y| \equiv 0 \bmod k$, on the other hand $|y|=k-i+|x|+j$, hence $i \equiv j+|x| \bmod k$. 
Note that, two words from $\Sigma^{k} \backslash S_{k}$ overlap only in case of $v$ and $u$. More precisely, two last letters of $v$ overlap with first two letter of $u$ leading to the word $b^{k-1} a^{k-1}$. The following statement can be easily proved using the same argument as in the previous lemma.

Lemma 4. Let $v$ and $u$ be two consecutive overlapping occurrences in a word $w$, and let $F_{v}$ and $F_{u}$ be the corresponding sets of forbidden positions. Then

$$
F_{v} \subseteq\left\{j-2 \bmod k \mid j \in F_{u}\right\} \cup\{0\} .
$$

Previous lemmas allow us to make the following observation. Let $p, q \in\{u, v\}$ be two consecutive occurrences in $w$. Then forbidden positions in $p$ except 0 are inherited from forbidden positions in $q$, and position 0 may appear in $F_{p}$ according to lemma 2, In our proofs we will trace backwards forbidden positions only in occurrences of words from $\Sigma^{k} \backslash S_{k}$ starting from the last one. Besides, the number of forbidden positions in consecutive occurrences increases by at most 1 .

\section{$3 \quad$ Upper bound for $u w l\left(S_{k}\right)$}

In this section we prove that the set $S_{k}$ is non-complete by presenting an uncompletable word $w$ of length $5 k^{2}-17 k+13$ for $k \geq 4$.

Theorem 1. For $k \geq 4$ the set $S_{k}$ is not complete and there exists an uncompletable word of length $5 k^{2}-17 k+13$.

Proof. For clarity by $r$ we denote overlapping occurrences of $v$ and $u$, i.e. $r=$ $b^{k-1} a^{k-1}$. Consider the word $\omega=u \cdot \prod_{i=1}^{k-3}\left(r a^{i} \cdot b^{k-2-i} r.\right) v$, where c can be replaced by any letter of the alphabet $\stackrel{i=1}{\Sigma}$. Let us enumerate occurrences of $v$ counting backwards from the last one (the first occurrence of $v$ in this order will have number 0 ), and let $F_{v}^{i} \subseteq\{0, \ldots, k-1\}$ be the set of forbidden positions in the $i$ th occurrence of $v$. Occurrences of $u$ are counted in the same way (but starting from 1 instead of 0 ) and $F_{u}^{i}$ are defined analogously. Note that, $F_{v}^{0}=\{0\}$. Then using previous lemmas it is easy to see that $F_{u}^{1}=\{1\}$ and $F_{v}^{1}=\{0, k-1\}$. By lemma 3 we have $F_{u}^{2} \subseteq\{0, k-2, k-1\}$ and by lemmas 1 and 2 we get $F_{u}^{2}=\{0, k-2, k-1\}$. Analogously we obtain $F_{v}^{2}=\{0, k-3, k-2\}$. Now assume $F_{v}^{2 i}=\{0, k-i-2, \ldots, k-2\}, 1 \leq i \leq k-4$, and let us show that $F_{v}^{2(i+1)}=\{0, k-i-3, \ldots, k-2\}$. Applying step by step lemmas 14 we obtain the following sets of forbidden positions:

$$
\begin{aligned}
& F_{u}^{2 i+1}=\{0,1, k-i, \ldots, k-1\}, \\
& F_{v}^{2 i+1}=\{0, k-i-1, \ldots, k-1\}, \\
& F_{u}^{2 i+2}=\{0, k-i-2, \ldots, k-1\}, \\
& F_{v}^{2 i+2}=\{0, k-i-3, \ldots, k-2\} .
\end{aligned}
$$

Thus, for $i=k-3$ we have $F_{v}^{2(k-3)}=\{0,1, \ldots, k-2\}$. Hence the set of forbidden positions in the last occurrence of $u$ is $F_{u}^{2(k-3)+1}=\{0,1, \ldots, k-1\}$, which means that the word $\omega$ is uncompletable. Its length equals $5 k^{2}-17 k+13$. 


\section{Lower bound for $u w l\left(S_{k}\right)$}

First we prove some nice properties of a minimal uncompletable word in $S_{k}$.

Theorem 2. Consider a minimal uncompletable word $w$. Then $u$ is a prefix of $w$ and $v$ is its suffix.

Proof. The word $w$ has either $u$ or $v$ as a factor, otherwise $w \in \operatorname{Pref}\left(\left(S_{k} \cap \Sigma^{k}\right)^{*}\right)$. Let $w=w^{\prime} x$, where $|x|=k$, and suppose $x \neq v$. Let $x=x^{\prime} z$, where $z \in \Sigma$. Since $w$ is minimal, we conclude that $w^{\prime} x^{\prime} \in \operatorname{Fact}\left(S_{k}^{*}\right)$, which means $r w^{\prime} x^{\prime}=q y$, for some $q \in S_{k}^{*}$ and $|y| \leq k-1$. If $|y|<k-1$, then $y z \in \operatorname{Pref}\left(S_{k}\right)$, because all the words of length at most $k-1$ are prefixes of some words in $S_{k}$. If $|y|=k-1$, then $y z=x$. If $x \neq u$, then $y z \in S_{k}$, and $q y z=r w \in S_{k}^{*}$. If $x=u$, then $y=b a^{k-2}$, $z=a$ and we have for instance $r w a^{k-1} \in S_{k}^{*}$. In any case we get a contradiction with the fact that $w$ is uncompletable. Thus, $w$ has $v$ as a suffix.

Now we are going to investigate one particular symmetry property of uncompletable words for $S_{k}$. It is trivial that the mirror image $\overleftarrow{S}$ of a non-complete set $S$ is again non-complete. Moreover, mirror images $\overleftarrow{w}$ of uncompletable words $w$ for $S$ are uncompletable for $\overleftarrow{S}$. The same property holds true for renaming morphism: $\varphi(a)=b$ and $\varphi(b)=a$. Applying these statements to our set we get $T_{k}=\overleftarrow{S_{k}}=\varphi\left(S_{k}\right)$, where

$$
T_{k}=\left(\Sigma^{k} \backslash\left\{a^{k-1} b, a b^{k-1}\right\}\right) \cup\left(\Sigma^{k-1} \backslash\left\{a^{k-1}, b^{k-1}\right\}\right) .
$$

So, if $w$ is an uncompletable word for $S_{k}$, then $\varphi(\overleftarrow{w})$ is also uncompletable for $S_{k}$. As we have already shown, every minimal uncompletable word has $v$ as a suffix. From the symmetry property it follows that every such word has $u$ as a prefix.

The suffix $v$ of a minimal uncompletable word $w$ has only one forbidden position, namely 0 , and in the prefix $u$ of $w$ all the positions from 0 to $k-1$ are forbidden. Thus, we have to analyze how forbidden positions change from one occurrence of a word from $\Sigma^{k} \backslash S_{k}$ to the next one.

Consider an arbitrary occurrence of a word from $\Sigma^{k} \backslash S_{k}$ in $w$. Let $F$ be the set of its forbidden positions. We will make use of the following representation of $F$ :

$$
F=\left[f_{1,1}, f_{1,2}, \ldots, f_{1, m_{1}} ; f_{2,1}, \ldots, f_{2, m_{2}} ; \ldots ; f_{n, 1}, \ldots, f_{n, m_{n}}\right]
$$

where $f_{i, j+1} \equiv f_{i, j}+1 \bmod k, f_{i+1,1}>f_{i, m_{i}}$ and $n \geq 1$. Simply speaking, we partition the set $F$ into blocks of consecutive (with respect to cyclic order) forbidden positions.

Example 2. Consider the word $b a^{10}$, and let $F=\{0,1,2,5,6,8,10\}$ be the set of its forbidden positions with respect to $S_{11}$. Then according to our representation $F=[5,6 ; 8 ; 10,0,1,2]$.

Theorem 3. Let $p$ and $q$ be two consecutive occurrences of words from $\{u, v\}$ in a minimal uncompletable word $w$. Let $F_{p}$ and $F_{q}$ be the sets of forbidden positions in $p$ and $q$ respectively. If $\left|F_{p}\right|>\left|F_{q}\right|$, then one of the following holds true: 
(i) $p=q=u, F_{p}=\{0, k-j, \ldots, k-1\}$ and $F_{q}=\{1, \ldots, j\}$, where $1 \leq j \leq k-2$;

(ii) $p=v, q=u$, these occurrences overlap, $F_{p}=\{0, k-1\}$ and $F_{q}=\{1\}$;

(iii) $p=u, q=v, F_{p}=\{0, j-i-1, \ldots, k-1\}, F_{q}=\{0, \ldots, i, j, \ldots, k-1\}$, where $j \not \equiv i+1 \bmod k$ and there are $k-1-i \bmod k$ letters between these occurrences;

Proof. Let $F_{q}=\left[f_{1,1}, \ldots, f_{1, m_{1}} ; \ldots ; f_{n, 1}, \ldots, f_{n, m_{n}}\right]$. First assume that $p$ and $q$ do not overlap, so let $p x q$ be the corresponding factor of $w$.

Case 1. Let $p=q=u$. Consider the case $n \geq 2$. Then there exists $1 \leq i \leq n$ such that $f_{i, 1} \geq 2$. If $f_{i, 1}>2$, then by lemma 1 position $f_{i, 1}+|x| \notin F_{p}$. If $f_{i, 1}=2$, then by the same lemma $f_{i, 1}+|x| \in F_{p}$ implies $x=x^{\prime} b^{k-2}$. But then $v$ is a factor of $p x q$ which contradicts the fact that $p$ and $q$ are two consecutive occurrences from $\{u, v\}$. Thus, $f_{i, 1}+|x|$ is not forbidden in $p$. From lemma 3 it follows that $F_{p} \subseteq\left\{j+|x| \bmod k \mid j \in F_{q}\right\} \cup\{0\}$, whence $\left|F_{p}\right| \leq\left|F_{q}\right|$, a contradiction. Consequently $n=1$ and $F_{q}=\left[f_{1,1}, \ldots, f_{1, m}\right]$. If $f_{1,1} \geq 2$, then following the same argument as above, we conclude that $\left|F_{p}\right| \leq\left|F_{q}\right|$, hence $f_{1,1} \in\{0,1\}$. Note that, if $f_{1,1}=0$, by lemma 2 we have $k-1 \in F_{q}$. It means that $F_{q}=\{0,1, \ldots, k-1\}$ which contradicts minimality of $w$. Thus $f_{1,1}=1$. If $f_{1, m}=k-1$, then by lemma 2 we obtain that $0 \in F_{q}$ and in this case $f_{1,1}$ cannot be equal to 1 . So $f_{1, m} \leq k-2$. Now it remains to prove that $F_{p}$ has form as stated in $(i)$. Since $\left|F_{p}\right|>\left|F_{q}\right|$, we have $0 \in F_{p}$. Then by lemma 2 we obtain that $k-1$ is also in $F_{p}$. So there exists a position $i \in F_{q}$ satisfying $i+|x| \equiv k-1$ $\bmod k$. If $i<f_{1, m}$, then $i+1+|x| \equiv 0 \bmod k$, hence $\left|F_{p}\right| \leq\left|F_{q}\right|$. Thus $i=f_{1, m}$, $|x| \equiv k-f_{1, m}-1 \bmod k$ and by lemma 3 we deduce $F_{p}=\left\{0, k-f_{1, m}, \ldots, k-1\right\}$.

Case 2. Let $p=v, q=u$. We are to show that either $f_{1,1}+|x|$ is not forbidden in $p$ or $f_{1,1}+|x| \equiv 0 \bmod k$, so in both cases $\left|F_{p}\right| \leq\left|F_{q}\right|$. It holds for $f_{1,1} \geq 2$ by the argument as in the previous case. Note that, $f_{1,1} \neq 0$, otherwise by lemma 2 position $k-1 \in F_{q}$ which contradicts our representation of a set of forbidden positions. Therefore, assume $f_{1,1}=1$. Suppose $1+|x| \equiv i \bmod k$. If $0<i<k-1$, then by lemma 1 position $i$ is not forbidden in $p$. If $i=0$, then lemma 3 implies $\left|F_{p}\right| \leq\left|F_{q}\right|$. If $i=k-1$, then by lemma 1 we have $i \in F_{p}$ only if $a^{k-2}$ is a prefix of $x$, but then $p$ and $q$ are not consecutive occurrences from $\{u, v\}$.

Case 3. Let $p=u, q=v$. Suppose $n \geq 2$, and consider arbitrary positions $f_{i, 1}<f_{j, 1} \in F_{q}$. We show that either $f_{i, 1}+|x| \bmod k \notin F_{p}$ or $f_{j, 1}+|x|$ $\bmod k \notin F_{p}$. Arguing by contradiction, suppose both positions are forbidden in $p$. Then by lemma 1 the word $x$ must have suffix $b^{k-f_{j, 1}}$ (if $|x|<k-f_{j, 1}$, then $f_{j, 1}+|x| \bmod k \notin F_{p}$ by lemma 1). Analogously $b^{k-f_{i, 1}}$ must be the suffix of $x$, and if $|x|<k-f_{i, 1}$, then $f_{i, 1}+|x| \bmod k \notin F_{p}$ by lemma 11 But then by the same lemma $k+\left(k-f_{j, 1}\right)$ last letters of $x$ are $b$ 's. Continuing this argument we get that $\ell k-f_{j, 1}$ last letters of $x$ are $b$ 's for any positive integer $\ell$. It means that there exists no finite word $x$ such that both positions $f_{i, 1}+|x| \bmod k$ and $f_{j, 1}+|x| \bmod k$ are forbidden in $p$. Hence $n=1$, and $F_{q}=\left[f_{1,1}, \ldots, f_{1, m}\right]$. Since $\left|F_{p}\right|>\left|F_{q}\right|$, by lemmas 3 and 2 there is no $j$ such that $f_{1, j}+|x| \equiv 0 \bmod k$. Besides $f_{1, i}+|x| \equiv k-1 \bmod k$ for some $i$. If $i<m$, then $f_{1, i+1}+|x| \equiv 0$ $\bmod k$, which is impossible. Thus, $i=m$. Moreover, $|x| \equiv k-1-f_{1, m} \bmod k$. 
Since 0 is always forbidden in $v$, we can represent $F_{q}$ as $\{0, \ldots i, j, \ldots, k-1\}$ for some $0 \leq i<j \leq k-1$. Then by lemma 3 we have $F_{p}=\{0, j-i-1, \ldots, k-1\}$.

Case 4. Let $p=v, q=v$. If $f_{1,1}+|x| \notin F_{p}$ then $\left|F_{p}\right| \leq\left|F_{q}\right|$. Otherwise by lemma 1 either $f_{1,1}+|x| \equiv 0 \bmod k$ or $f_{1,1}+|x| \equiv k-1 \bmod k$. In the first case by lemma 3 we get $\left|F_{p}\right| \leq\left|F_{q}\right|$. In the latter case by lemma 11 the word $a^{k-2}$ have to be the prefix of $x$, which contradicts the fact that $p$ and $q$ are consecutive occurrences.

Now assume that $p=v, q=u$, and they overlap. If there exists $i$ such that $f_{i, 1}>2$ then by lemmas 1 and 4 position $f_{i, 1}-2 \notin F_{p}$, therefore $\left|F_{p}\right| \leq$ $\left|F_{q}\right|$. Note that, $f_{1,1} \neq 0$, otherwise by lemma 2 position $k-1 \in F_{q}$ which contradicts our representation of a set of forbidden positions. Hence, for all $i$ we have $f_{i, 1} \in\{1,2\}$. It immediately implies that $n=1$. If $f_{1, j}=2$ for some $j$ then by lemma 4 we get that $f_{1, j}-2$ is equal to 0 and $\left|F_{p}\right| \leq\left|F_{q}\right|$. Thus $F_{q}=\{1\}$ and $F_{p}=\{0, k-1\}$.

Lemma 5. Let $p$ and $q$ be two consecutive occurrences of $u$ in a word $w, F_{p}$ and $F_{q}$ be the corresponding sets of forbidden positions. If $F_{q}=\{0, i, \ldots, k-1\}$ and $\left|F_{p}\right|=\left|F_{q}\right|$, then $F_{p}=F_{q}$.

Proof. If $i=1$, then the statement of lemma obviously holds true. So we may assume $i>1$. Let $p x q$ be the factor of $w$. Then by lemma 3 we have $F_{p} \subseteq\{0,|x|$ $\bmod k, i+|x| \bmod k, i+1+|x| \bmod k, \ldots, k-1+|x| \bmod k\}$. By lemma 1 position $i+|x| \bmod k \notin F_{p}$. Thus in order to have $\left|F_{p}\right|=\left|F_{q}\right|$, it is necessary that $0 \in F_{p}$ and $j+|x| \not \equiv 0 \bmod k$ for all $i+1 \leq j \leq k$. Lemma 2 implies $k-1 \in F_{p}$. It means that $j+|x| \equiv k-1 \bmod k$ for some $i+1 \leq j \leq k$. It is straightforward that $j=k,|x| \equiv k-1 \bmod k$ and $F_{p}=\{0, i, i+1, \ldots, k-1\}=F_{q}$.

Lemma 6. Let $p$ and $q$ be consecutive occurrences of $v$ and $u$ respectively in $a$ minimal uncompletable word $w$. Let $F_{p}$ and $F_{q}$ be the corresponding sets of forbidden positions. If $F_{q}=\{0, i, \ldots, k-1\}$ and $\left|F_{p}\right|=\left|F_{q}\right|$, then these occurrences overlap and $F_{p}=\{0, i-1, \ldots, k-2\}$.

Proof. If $i=1$, then trivially $w$ is not a minimal uncompletable word, so $i>1$. Suppose $p$ and $q$ do not overlap, so there exists $x$ such that $p x q$ is a factor of $w$. By lemma 3 we have $F_{p} \subseteq\{0,|x| \bmod k, i+|x| \bmod k, i+1+|x| \bmod k, \ldots, k-$ $1+|x| \bmod k\}$ and from lemma 1 it follows that either $i+|x| \notin F_{p}$ or $i+|x| \equiv 0$ $\bmod k$. Moreover, if $j+|x| \equiv 0 \bmod k$ for some $i+1 \leq j \leq k$, then we immediately get $\left|F_{p}\right|<\left|F_{q}\right|$. So we may assume that $j+|x| \not \equiv 0 \bmod k$ for all $i+1 \leq j \leq k$. First let $|x|>k-(i+1)$. Then by lemma 1 if position $i+1+|x| \bmod k$ is forbidden in $p$, then either $i+1+|x| \equiv 0 \bmod k$ or $i+1+|x| \equiv k-1 \bmod k$. The first case contradicts our assumption. In the latter case $x=a^{k-2} x^{\prime}$, but this contradicts the fact that $p$ and $q$ are consecutive occurrences. Thus, both cases are impossible. So $0 \leq|x| \leq k-(i+1)$, but then we have $i+1 \leq j=k-|x| \leq k$. It means that $j \bmod k$ is a forbidden position in $q$ and $j+|x| \equiv 0 \bmod k$, which again contradicts our assumption that $j+|x| \not \equiv 0$ $\bmod k$ for all $i+1 \leq j \leq k$. Therefore occurrences $p$ and $q$ overlap. By lemmas 1 and 4 we get $F_{p}=\{0, i-1, \ldots, k-2\}$. 
Lemma 7. Let $p$ and $q$ be two consecutive occurrences of $v$ in a minimal uncompletable word $w$, and let $F_{p}$ and $F_{q}$ be the corresponding sets of forbidden positions. If $F_{q}=\{0, i, \ldots, k-2\}$ then $\left|F_{p}\right|<\left|F_{q}\right|$.

Proof. Consider the factor $p x q$ of $w$. By lemma 3 we get $F_{p} \subseteq\{0,|x| \bmod k, i+$ $|x| \bmod k, i+1+|x| \bmod k, \ldots, k-2+|x| \bmod k\}$. We are going to show that $|x| \bmod k$ is not forbidden in $p$. From lemma 1 it trivially follows that the position $|x| \bmod k \in F_{p}$ if either $|x| \equiv 0 \bmod k$ or $|x| \equiv k-1 \bmod k$. The first case contradicts minimality of $w$, for we would have $F_{p}=F_{q}$. In the latter case by the same lemma we conclude $x=a^{k-2} x^{\prime}$, which contradicts the fact that $p$ and $q$ are consecutive occurrences. Similar arguments can be applied to $i+|x| \bmod k$. Namely, if $i+|x| \equiv k-1 \bmod k$, then again $x=a^{k-2} x^{\prime}$, a contradiction. So either $i+|x| \equiv 0 \bmod k$ or $i+|x| \bmod k \notin F_{p}$. In both cases we have $\left|F_{p}\right|<\left|F_{q}\right|$.

Lemma 8. Let $p$ and $q$ be consecutive occurrences of $u$ and $v$ respectively in a word $w$. Let $F_{p}$ and $F_{q}$ be the corresponding sets of forbidden positions, and let $\left|F_{p}\right|=\left|F_{q}\right|$. If $F_{q}=\{0, i, \ldots, k-2\}$ with $i>1$ and $F_{p}=\left[f_{1,1}, \ldots, f_{1, m}\right]$, then either $F_{p}=\{0,1, i+2, i+3, \ldots, k-1\}$ or $F_{p}=\{0, i+1, i+2, \ldots, k-1\}$.

Proof. Consider the factor $p x q$ of $w$. By lemma 3 we get $F_{p} \subseteq\{0,|x| \bmod k, i+$ $|x| \bmod k, i+1+|x| \bmod k, \ldots, k-2+|x| \bmod k\}$. Arguing as in case 3 of the theorem 3 we easily obtain that either $|x| \bmod k \notin F_{p}$ or $i+|x| \bmod k \notin F_{p}$. Suppose that position $|x| \bmod k$ is not forbidden in $F_{p}$. Since $\left|F_{p}\right|=\left|F_{q}\right|$ it is necessary that $0 \in F_{p}$, therefore $k-1$ is also in $F_{p}$ by lemma2. Every $i \leq j \leq k-3$ satisfies $j+|x| \not \equiv k-1 \bmod k$, since otherwise we would have $j+1+|x| \equiv 0$ $\bmod k$, which would imply $\left|F_{p}\right|<\left|F_{q}\right|$. Therefore, $k-2+|x| \equiv k-1 \bmod k$, whence $|x| \equiv 1 \bmod k$ and $F_{p}=\{0, i+1, \ldots, k-1\}$. Assume now that position $i+|x| \bmod k \notin F_{p}$. Following the same argument as in the previous case, we get $k-1 \in F_{p}$ and either $|x| \equiv k-1 \bmod k$ or $k-2+|x| \equiv k-1 \bmod k$. In the first case $F_{p}=\{0, i, \ldots, k-3, k-1\}$, and it has more than one block of consecutive forbidden positions, which contradicts the statement of lemma. In the latter case $|x| \equiv 1 \bmod k$ and $F_{p}=\{0,1, i+2, \ldots, k-1\}$.

Theorem 4. The length of a minimal uncomletable word for $S_{k}$ is at least $5 k^{2}-$ $17 k+13$ for $k \geq 4$.

Proof. Let $w$ be an arbitrary minimal uncomletable word for $S_{k}$. By theorem 2 the word $u$ is a prefix of $w$ and $v$ is its suffix. Note that, we have $F_{u}=\{0,1, \ldots, k-1\}$ and $F_{v}=\{0\}$ in the aforementioned occurrences of $u$ and $v$.

If $s$ and $t$ are two consecutive occurrences from $\{u, v\}$ such that $\left|F_{s}\right|>$ $\left|F_{t}\right|$, then we say that $s$ is an increasing occurrence. Recall that by lemma 3 it means that $\left|F_{s}\right|=\left|F_{t}\right|+1$. Since there is only one forbidden position in the last occurrence of $v$ in $w$ and $k$ forbidden positions in the first occurrence of $u$, there must be at least $k-1$ increasing occurrences in $w$. Now we are going to estimate the length of a factor between two consecutive such occurrences. 
Consider a factor $p x q$ of $w$ such that $p$ and $q$ are the only increasing occurrences inside this factor. Note that, for an occurrence $r \in\{u, v\}$ in $p x q$, different from $p$ and $q$ (if any), $\left|F_{r}\right|=\left|F_{q}\right|$. Otherwise $p$ and $q$ are not consecutive increasing occurrences.

Let $3 \leq\left|F_{q}\right|<\left|F_{p}\right| \leq k$. Then by theorem 3 we have $p=q=u, F_{q}=$ $\{0, i, \ldots, k-1\}$ and $F_{p}=\{0, i-1, \ldots, k-1\}$. Moreover, $p$ and $q$ are not the only occurrences from $\{u, v\}$ in $p x q$. Assume first $i>2$, i.e. $\left|F_{p}\right|<k$.

Suppose that there is only one occurrence $r \in\{u, v\}$ in $p x q$ different from $p$ and $q$. Then from lemma 5 it follows that $r=v$ and since $\left|F_{r}\right|=\left|F_{q}\right|$, applying lemma 6 we obtain $F_{r}=\{0, i-1, \ldots, k-2\}$. But then since $i>2$ the set $F_{r}$ does not have the form required by theorem 3 for the condition $\left|F_{p}\right|>\left|F_{r}\right|$ to hold true.

Assume now that $r_{1}, r_{2} \in\{u, v\}$ are the two occurrences in $p x q$ different from $p$ and $q$. By the same argument as above $r_{2}=v$ and $F_{r_{2}}=\{0, i-1, \ldots, k-2\}$. If $r_{1}=u$, then on the one hand by theorem 3 it should be $F_{r_{1}}=\{1,2, \ldots, k-$ $i+1$ \}. On the other hand by lemma 8 position 0 have to be forbidden in $F_{r_{1}}$, a contradiction. If $r_{2}=v$, then by lemma 7 we have $\left|F_{r_{1}}\right|<\left|F_{r_{2}}\right|$, which is impossible. So there are at least three occurrences of words from $\{u, v\}$ in $p x q$ except $p$ and $q$.

Suppose that $r_{1}, r_{2}, r_{3} \in\{u, v\}$. As we have already seen before $r_{3}=v$ and $F_{r_{3}}=\{0, i-1, \ldots, k-2\}$. It immediately follows from lemma 7 that $r_{2}=u$. Then $F_{r_{2}}$ is either $\{0,1, i+1, i+2, \ldots, k-1\}$ or $\{0, i, i+1, \ldots, k-1\}$ by lemma 8 , The latter case contradicts minimality of $w$, since we would have $\left|F_{r_{2}}\right|=\left|F_{q}\right|$. Assume $r_{1}=u$, then by theorem 3 we have $F_{r_{1}}=\{1,2, \ldots, k-i+1\}$. Let $r_{1} y r_{2}$ be a factor of $p x q$. Note that, $0 \notin F_{r_{1}}$ and by lemma 1 position $i+1+|y|$ $\bmod k \notin F_{r_{1}}$. Thus $\left|F_{r_{1}}\right|<\left|F_{r_{2}}\right|$, a contradiction. Therefore $r_{1}=v$. This case is possible and exactly this situation takes place in the word presented in theorem 1 .

Now we are going to estimate the length of $x$ in this case. By lemma 6 the factor $r_{3}$ overlaps with $q$, so we can factorize $x$ in the following way: either $x=y_{1} r_{1} y_{2} r_{2} y_{3} b^{k-2}$ or, if $r_{1}$ and $r_{2}$ overlap, $x=z_{1} b^{k-1} a^{k-1} z_{2} b^{k-2}$. By the proof of lemma 8 , since $F_{r_{2}}=\{0,1, i+1, i+2, \ldots, k-1\}$, we have $\left|y_{3}\right| \equiv\left|z_{2}\right| \equiv 1$ mod $k$. Let us first estimate the length of $z_{1}$. Since $r_{1}$ and $r_{2}$ overlap, lemma 4 implies $F_{r_{1}}=\{0, i, i+1 \ldots, k-1\}$. Then by theorem 3 there are $k-1 \bmod k$ letters between $p$ and $r_{1}$, thus $\left|z_{1}\right| \equiv k-1 \bmod k$. So in this case we have $|x| \geq 4 k-4$. Now let us assume that $r_{1}$ and $r_{2}$ are not overlapping. By theorem 3 the factor $r_{1}$ must have only one block of consecutive forbidden positions. By lemma 3 we have $F_{r_{1}} \subseteq\left\{0, i+1+\left|y_{2}\right| \bmod k, i+2+\left|y_{2}\right| \bmod k, \ldots, k-1+\left|y_{2}\right|\right.$ $\left.\bmod k,\left|y_{2}\right| \bmod k,\left|y_{2}\right|+1 \bmod k\right\}$. Note that, by lemma 1 either $i+1+\left|y_{2}\right|$ $\bmod k \notin F_{r_{1}}$ or $i+1+\left|y_{2}\right| \equiv 0 \bmod k$. So for the set $F_{r_{1}}$ to have only one block of consecutive forbidden positions and the same cardinality as $F_{r_{2}}$ we must have either $1+\left|y_{2}\right| \equiv k-1 \bmod k$ or $i+2+\left|y_{2}\right| \equiv 1 \bmod k$. In the first case we have $\left|y_{2}\right| \equiv k-2 \bmod k$ and $F_{r_{1}}=\{0, i, i+1, \ldots, k-1\}$, but this is the same as in the case of overlapping occurrences $r_{1}$ and $r_{2}$, so this is impossible in a minimal uncompletable word. In the second case we have $\left|y_{2}\right| \equiv k-i-1 \bmod k$ 
and $F_{r_{1}}=\{0,1,2, \ldots, k-i\}$. Then by theorem 3 we conclude that $\left|y_{1}\right| \equiv i-1$ $\bmod k$. Therefore in this case we have $|x| \geq 4 k-3$.

It is not hard to see that, if there are more than 3 occurrences from $\{u, v\}$ different from $p$ and $q$ in $p x q$, then even if some of them overlap, the total length of the word $x$ is at least $4 k-4$. So we conclude that $|x| \geq 4 k-4$. Note that, $|x|=4 k-4$ for the word from theorem 1 .

Now let $i=2$, i.e. $F_{p}=\{0,1, \ldots, k-1\}$ and $F_{q}=\{0,2,3, \ldots, k-1\}$. Arguing as above, by lemmas 5 and 6 there is an occurrence $r=v$ just before $q$, overlapping with $q$ and $F_{r}=\{0,1, \ldots, k-2\}$. If this is the only occurrence and $p x q=p y b^{k-2} q$, then by theorem 3 we have $|y| \equiv 1 \bmod k$ and $F_{p}=$ $\{0,1, \ldots, k-1\}$, so here we have $|x| \geq k+1$. More occurrences from $\{u, v\}$ inside $p x q$ will obviously give a longer factor $x$.

The previous argument implies that any minimal uncompletable word has prefix $p y b^{k-2} q$ with $p=q=u$ and $|y| \equiv 1 \bmod k$. From the symmetry property observed in theoren 2 we deduce that any minimal uncompletable word has suffix $q^{\prime} a^{k-2} \hat{y} p^{\prime}$ with $p^{\prime}=q^{\prime}=v$ and $|\hat{y}| \equiv 1 \bmod k$. Clearly $F_{p^{\prime}}=\{0\}$. To calculate $F_{q^{\prime}}$ note that, there is an occurrence $r=u$ overlapping with $q^{\prime}$ and $F_{r}=\{1\}$. From theorem 3 we deduce that $q^{\prime}$ is an increasing occurrence and $F_{q^{\prime}}=\{0, k-$ $1\}$. Let $p$ be the next increasing occurrence. If $\left|F_{p}\right|=3$, then the same theorem implies that $p=u, F_{p}=\{0, k-2, k-1\}$ and there are at least $k-1$ letters between $p$ and $q^{\prime}$. If $\left|F_{p}\right| \leq 2$, then we would obviously require at least $k$ letters between $q^{\prime}$ and an increasing occurrence with three forbidden positions.

Thus to increase the number of forbidden positions from 1 in the suffix $v$ of $w$ to 2 we need at least $k-1$ letters; from 2 to 3 - at least $k-1$; from $\ell$ to $\ell+1$ for $3 \leq \ell \leq k-2$ we need at least $4 k-4$ letters, and finally, from $k-1$ to $k$ we need $k-1$ letters. Besides we have at least $k-1$ increasing occurrences and the suffix $v$ of $w$ with only one forbidden position. Thus the length of a minimal uncompletable word is at least $3(k-1)+(4 k-4)(k-4)+k(k-1)+k=$ $5 k^{2}-17 k+13$.

\section{Conclusion}

The series of sets $S_{k}$ was found during exhaustive computational experiment. We searched for maximal with respect to inclusion non-complete sets among all the subsets of $\Sigma^{\leq 3}$; we were interested in such sets having longest possible minimal uncompletable word. We have found two extreme sets up to renaming letters and taking mirror image. Namely, $S_{3}=\left(\Sigma^{3} \backslash\{b a a, b b a\}\right) \cup\left(\Sigma^{2} \backslash\{a a, b b\}\right)$ and $\left(\Sigma^{3} \backslash\{b a a, b b a\}\right) \cup\left(\Sigma^{2} \backslash\{a b, b a\}\right)$. Computation was based on representation of a set $S^{*}$ as a flower automaton and on the fact that $S$ is non-complete if and only if the corresponding non-deterministic automaton is synchronizing. Moreover, the set of uncompletable words coincides with the language of synchronizing words, see [1] and 3] for more details. The same task for $k=4$ was unfeasible for a typical laptop, so the search was performed with restriction $\left|\Sigma^{4} \cap S\right| \geq 11$. There is only one extreme non-complete set up to renaming letters and taking mirror image in this class. Namely, $\left(\Sigma^{4} \backslash\{a a b b, a b a a, a b b b\}\right) \cup$ 
$\left(\Sigma^{3} \backslash\{a b a, b b a, b b b\}\right)$. The length of a minimal uncompletable word for this example is 31 compared to 25 for the set $S_{k}$. So $S_{k}$ is not optimal even for $k=4$. Thus, the lower bound $5 k^{2}-17 k+13$ is likely to be improved. Nevertheless, the most interesting question whether the tight bound is quadratic remains open.

\section{References}

1. J. Berstel, D. Perrin, and C. Reutenauer. Codes and automata, Cambridge University press, 2009.

2. G. Fici, E. Pribavkina, J. Sakarovitch. On the Minimal Uncompletable Word Problem, CoRR, http://arxiv.org/abs/1002.1928, 2010.

3. E. V. Pribavkina. Slowly synchronizing automata with zero and incomplete sets, CoRR, http://arxiv.org/abs/0907.4576, 2009.

4. N. Rampersad, J. Shallit, Z. Xu The computational complexity of universality problems for prefxes, suffixes, factors, and subwords of regular languages, CoRR, http://arxiv.org/abs/0907.0159, 2009.

5. A. Restivo. Some remarks on complete subsets of a free monoid, Quaderni de "La ricerca scientifica", CNR Roma 109 (1981) 19-25. 